\title{
Designing Personalized Toe Spreaders for Hallux Valgus with Three-Dimensional Scanning and Printing
}

\author{
Yong Ho Cha1, Sung Jae Kim², Keun Ho Lee ${ }^{3}$, Jeong-Yi Kwon², Dong-Hyeon Kim4, Anna Seo4, Sang Jun \\ Kim $^{2}$ \\ 1Department of Physical Therapy, Samsung Medical Center \\ Gangnamgu, Irwonro, 50th, Seoul, 135-710 \\ 2Department of Physical and Rehabilitation Medicine, Samsung Medical Center \\ Sungkyunkwan University School of Medicine \\ Gangnam-Gu, Irwonro, 50th, Seoul, Republic of Korea, 135-710 \\ catedral.sjk@gmail.com \\ ${ }^{3}$ Department of Occupational Therapy, Samsung Medical Center \\ Gangnamgu, Irwonro, 50th, Seoul Korea, 135-710 \\ 43D Convergence Center, Kyungpook National University \\ Donggu, Dongnaero, 70th, Daegu, Korea, 41061
}

\begin{abstract}
The aim of this study is to make personalized toe spreaders for hallux valgus with $3 D$ scanning and printing with a low cost. Eight patients (14 feet) with hallux valgus participated in our study and were scanned with a $3 D$ scanner. After designing toe spreaders with a modeling program, we made toe spreaders using a $3 D$ printing technique with the selective laser sintering method. The hallux valgus angle (HVA) and intermetatarsal angle (IMA) were compared before and after wear. Changes in the HVA and IMA after wearing the toe spreader were significantly different $(P<0.001$ and $P=0.034)$. The total amount of time to make a customized toe spreader was approximately seven hours and the total price was approximately 50 dollars. Personalized toe spreaders for hallux valgus made with $3 D$ scanning and $3 D$ printing techniques improved the HVA and IMA. This suggests the possibility of the commercial use of $3 D$ printed customized toe spreaders for hallux valgus.
\end{abstract}

Keywords: Hallux Valgus, 3D Imaging, Medical Devices, Models, Structural, Personalized, Toe Spreader.

(C) Copyright 2018 Authors - This is an Open Access article published under the Creative Commons Attribution License terms (http://creativecommons.org/licenses/by/3.0). Unrestricted use, distribution, and reproduction in any medium are permitted, provided the original work is properly cited.

\section{Introduction and Related Work}

Hallux valgus is a foot deformity that is characterized by lateral deviation of the great toe and medial deviation of the first metatarsal; it is also known as a bunion, and the deformity of the great toe can cause pain. Its treatment consists of the use of toe spreaders, valgus splints, and operative correction.

Among the treatments, a toe spreader is a simple, cheap, and easy orthosis to wear. A toe spreader corrects the deformity when worn by separating the first from the second toes and can reduce the pain caused by the bunion by reducing the protrusion of the metatarsal head. A toe spreader can be worn everyday if it fits into the toe space of the patient, it can provide comfort to the patient, and it can correct the toe axis when worn. However, ready-made silicone toe spreaders do not fit accurately into the toe space of certain patients, and these ready-made silicone toe spreaders cannot correct the toe axis accurately because the deviation varies according to the patients. Custom-made toe spreaders can solve these problems but these toe spreaders take time to make; several days to a week and consequently expensive; about 150 dollars. They needs to be reconstructed from scratch if they are destroyed. In addition, if the shape of a 
patient's toe changes, the custom-made toe spreader will not fit, and it is difficult to modify the toe spreader.

The three-dimensional (3D) printing technique, also known as additive manufacturing, has been utilized in various medical fields. Its use can be broadly classified into four fields: patient education [1], surgical planning/simulation [2-4], artificial organs/implantable devices [5,6], and customized orthosis/prosthesis [7-9]. Compared to the traditional alternatives, the advantage of $3 \mathrm{D}$ printing is that the $3 \mathrm{D}$ printed products can be personalized, modified, and reproduced faster and more easily, which can lower the price of personalized products.

Therefore, if toe spreaders can be made using a 3D printer it will be beneficial in terms of correction of the toe axis, satisfaction, time, and costs because the toe spreaders are personalized, easily modifiable, and reproducible at a low price.

The purpose of this study is to produce personalized toe spreaders for hallux valgus using 3D printing and 3D scanning techniques and to evaluate the correction of the toe axis, satisfaction, time, and costs.

\section{Materials and Methods}

Volunteers were recruited who complained of a toe deformity and visited our outpatient department from February 2015 to May 2015. Among the volunteers, eight patients were selected who were diagnosed with hallux valgus. Hallux valgus was diagnosed when the hallux valgus angle (HVA) was less than $160^{\circ}$ according to an earlier study [10]. One patient had hallux valgus on his left foot, one patient had hallux valgus on the right foot, and six patients had hallux valgus on both feet. All patients had bunions on the first metatarsal head.

Two kinds of 3D scanners were used in this study to scan feet: Eva (Artec ${ }^{\mathrm{TM}}$ Eva, Artec Group, Luxembourg) and Spider (Artec ${ }^{\mathrm{TM}}$ Spider, Artec Group, Luxembourg). Eva and Spider have a similar video camera function in terms of the real-time capturing of objects. The scanner captures up to 16 frames per second and these frames are aligned automatically in real-time, which makes scanning easy and fast. With a $3 \mathrm{D}$ resolution of up to $0.5 \mathrm{~mm}$, Eva's textured models have been used in diverse industries, such as, computer graphics/animation, forensics, and medicine. Spider is designed to scan small objects with complex geometry, sharp edges and thin ribs. Therefore, Spider is usually used to scan small and complex objects, such as the human ear, keys, or coins.

The scanning process was conducted at our outpatient department. The 3D scanner could not detect the interdigital space because the interdigital space was shadowed; therefore, therapy putty (Sammons Preston $\AA$, Patterson Medical Holdings Inc., Illinois, USA) was inserted into the interdigital space between the first and second toes and the patients' feet were scanned in the inserted state with Eva (Figure 1). The size of the putty was modified until the axis of the first toe was less than $10^{\circ}$ from the axis of the first metatarsus (Figure 1). After extracting the putty from the foot, the molded putty was scanned again with Spider.

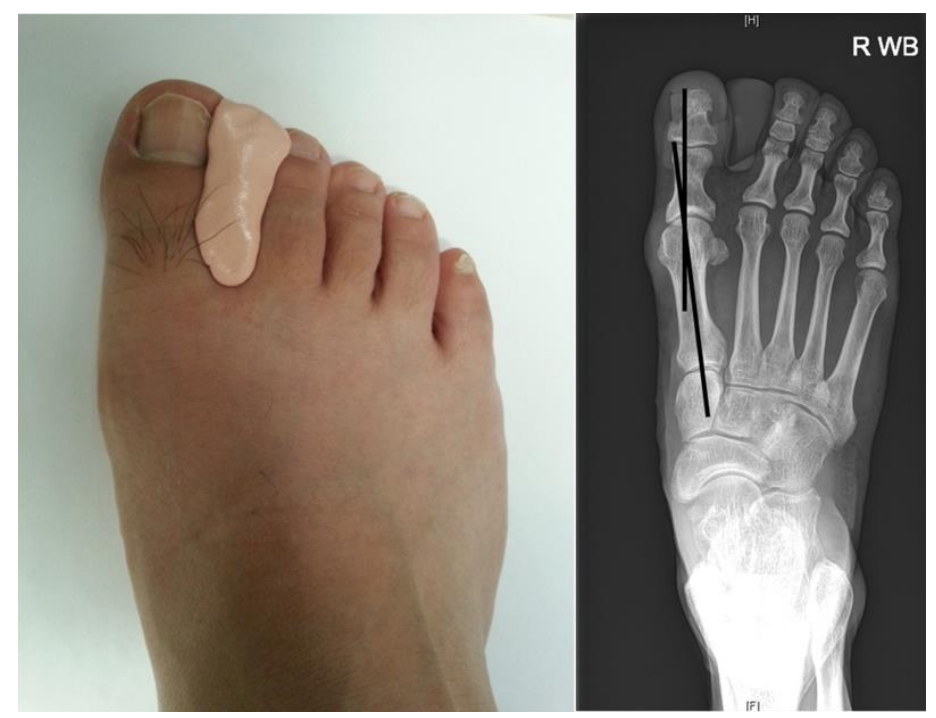

Figure 1. Therapy putty was inserted for scanning the interdigital space. The size of the putty was modified till the axis of first toe was less than $10^{\circ}$ along with the axis of the first metatarsus.

Using these scanned files, toe spreaders were designed with a modeling program (Geomagic Freeform, 3D SYSTEMS, SC, USA). This modeling program uses 'virtual clay' to provide unparalleled creative freeform, and includes a phantom device, a true 3D interface with force feedback. At this stage, a toe spreader was designed for each patient considering the shape of his/her foot (Figure 2). 


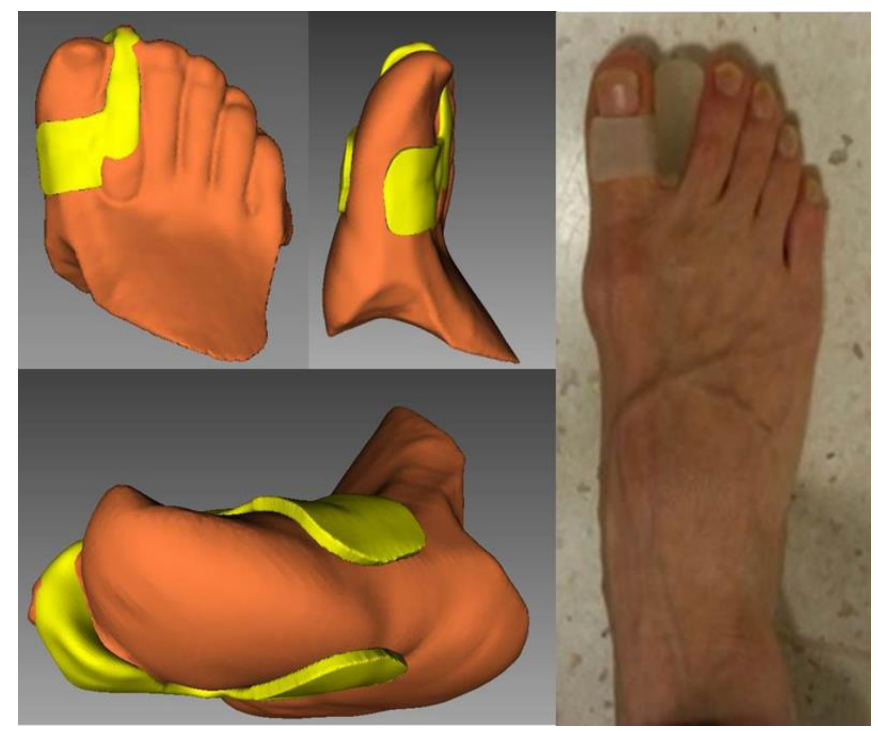

Figure 2. Toe spreader was designed with the modeling program. The toe spreader consisted of the separator part and the fixing part. Using these modeling files, the designed toe spreader was printed using selective laser sintering (SLS) type 3D printer and applied.

The newly designed toe spreader consisted of two components, the separator part and the fixing part. The separator part had a role in separating the first and second toes to bring the axis of the first toe and the first metatarsal within a $10^{\circ}$ range. The fixing component enveloped the first toe partially to inhibit displacement and rotation of the toe spreader.

Using these modeling files, the designed toe spreaders were printed using a selective laser sintering (SLS) type of 3D printer (Objet500 Connex1, Stratasys, USA). The printing process was conducted at the 3D Convergence Technology Center, Kyungpook National University. This printer offers 14 base materials including transparent materials with great dimensional stability and surface smoothness; rubber-like materials suitable for non-slip and non-scratch surfaces or simulation over molding; rigid opaque materials in white, gray and blue and black simulated polypropylene materials with toughness and durability to create living hinges, flexible closures and snap-fit prototypes. For preparation of the toe spreaders, VeroClear-RGD810 (Stratasys Ltd, Minnesota, USA) and TangoGray FLX950 (Stratasys Ltd, Minnesota, USA) were used. There was no post-processing after the toe spreader was printed out.

The personalized toe spreaders were received from the 3D Convergence Technology Center and were applied to the patients for one week and the evaluation was conducted after one week. For the evaluation, the Foot and Ankle Outcome Score (FAOS) [11] and User Satisfaction Survey (USS) [12] were used. In addition, after the toe spreaders were worn, simple radiographic anterior-posterior (AP) images of the foot in the standing position were checked for the measurement of the HVA and intermetatarsal angle (IMA). Image J software (NIH, USA) was used for the measurement of HVA and IMA.

For the statistical analysis, paired-t test was used to determine if there was a significant difference between the HVA and IMA before and after the application of the toe spreader and a Pearson correlation analysis was conducted to find the correlation between the initial severity of the angles and their changes. The necessary sample size was not calculated because this was a preliminary study. Statistical analyses were performed in SPSS 22.0 (IBM SPSS Statistics; IBM Corporation, Somers, NY).

Total elapsed time from scanning to manufacturing the products was measured. It consists of 3D scanning, $3 \mathrm{D}$ modeling, and printing out time. Total costs spent to make the 3D printed toe spreaders were measured. However, we did not consider the prices of 3D scanner, modeling program, and 3D printer.

Finally, to ensure the 3D-printed materials were safe, the possible side effects in patients including skin rash, dermatitis, and skin ulcer were investigated.

Written informed consent was obtained from all of the participants, and this research was approved by the Institutional Review Board of our hospital (IRB No.2014-12-013).

\section{Results}

All the data (14 feet among eight patients) were collected before and one week after the application of the toe spreaders. The initial HVA was $154.0 \pm 8.8^{\circ}$ and it changed to $160.5 \pm 7.8^{\circ}$ after wearing toe spreaders; this difference was statistically significant (Paired-t $t=-5.263$, $\mathrm{P}<0.001)$. The IMA also changed from $17.7 \pm 0.8^{\circ}$ to $16.5 \pm 1.9^{\circ}$ after wearing the toe spreader (Figure 3 ). 
HVA

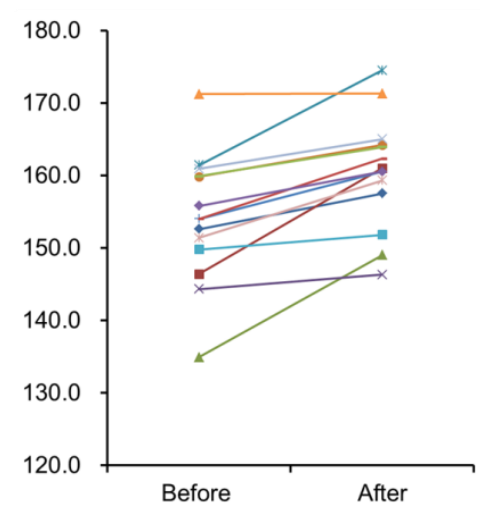

IMA

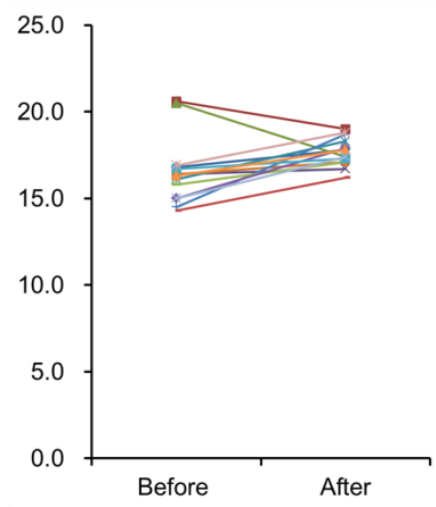

Figure 3. Changes in the hallux valgus angle (HVA) (left) and intermetatarsal angle (IMA) (right) after wearing the toe spreader were presented in 14 feet.

This difference was also statistically significant (Paired-t=-2.376, $P=0.034$ ). When the changes in the HVA and IMA were assessed according to their initial severity, the changes in the angle were correlated with the initial severity (Pearson $r=-0.480, P=0.043$ for HVA, Pearson $r=-0.905, P<0.001$ for IMA; Figure 4$)$. As the initial HVA and IMA angles were bigger, the changes in the HVA and IMA were bigger.
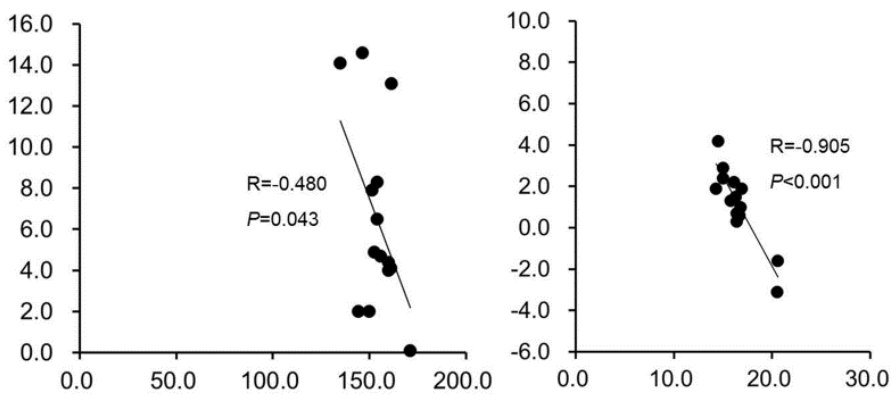

Figure 4. Changes in the HVA (left) and IMA (right) were assessed according to their initial severity in 14 feet.

The average score on the USS was $7.4 \pm 5.4$, and it ranged from 2 to 18 points. All scores for sub-items of the FAOS except for quality of life were more than 50 , which meant that all participants fully or generally felt satisfied. Three participants reported poor quality of life after wearing the toe spreaders because they felt uncomfortable during sports activities. All data from the USS and FAOS are presented in Table 1.

Table 1. Demographic data of patients and satisfaction scores.

\begin{tabular}{|c|c|c|c|c|c|c|c|c|}
\hline Patient & \multirow{2}{*}{ Sex } & \multirow{2}{*}{ Age } & USS & \multicolumn{5}{|c|}{ FAOS $^{\dagger}$} \\
\cline { 5 - 9 } & & & & Other & Pain & ADL & Sports & QoL \\
\hline JHK & F & 50 & 2 & 89.3 & 100 & 100 & 100 & 75 \\
\hline KIN & F & 65 & 18 & 96.4 & 94.4 & 72.1 & 95 & 18.6 \\
\hline HSL & F & 62 & 7 & 92.9 & 100 & 100 & 100 & 100 \\
\hline KSJ & F & 15 & 3 & 64.3 & 47.2 & 58.8 & 50 & 50 \\
\hline MOB & F & 55 & 7 & 89.3 & 80.6 & 97.1 & 100 & 43.8 \\
\hline JMY & F & 58 & 10 & 85.7 & 66.7 & 70.6 & 85 & 56.3 \\
\hline KKJ & F & 58 & 2 & 71.4 & 50 & 52.9 & 50 & 37.5 \\
\hline JMS & F & 50 & 10 & 100 & 100 & 53 & 50 & 56.3 \\
\hline
\end{tabular}

USS*: User satisfaction survey

FAOS†: Foot and ankle outcome score

The total amount of time to make a 3D printed personalized toe spreader was approximately seven hours. Elapsed time for 3D scanning was 30 minutes, time for designing was 1 hour, and time for printing was 5 hour and 30 minutes. The total cost for therapeutic putty and 3D printing materials used was approximately 50 dollars.

There was no case of skin rash, dermatitis, or skin ulcer during or after wearing the orthosis.

\section{Discussion}

We made personalized toe spreaders for hallux valgus by using 3D scanning and 3D printing techniques and applied these to patients with hallux valgus successfully. The HVA and IMA of patients improved significantly and patients were generally satisfied with these newly-designed toe spreaders. In addition, no side effects were detected.

We inserted putty into the interdigital space and positioned the axis of the first toe and first metatarsus in a single line. This might explain why the changes in the HVA were correlated with the initial severities of the HVA this resulted from more severe hallux valgus being corrected further by the toe spreader. Previously, ready-made toe spreaders were designed with limited shapes, which cannot modify the HVA individually unlike customized toe spreaders. 
From our study, the HVA and IMA changed by $6.5^{\circ}$ and $1.2^{\circ}$ on average, respectively and this finding was similar to that in the study by Tang et al [13], in which the HVA changed by $6.5^{\circ}$. Although this change was less than that after the surgical procedure, the first metatarsal osteotomy provides a correction on average of $20.1^{\circ}$ in a meta-analysis [14], and all participants had an HVA of more than $160^{\circ}$ except for one participant. A corrected HVA of more than $160^{\circ}$ could improve the biomechanical parts of the body, which could prevent possible complications including bunions, osteoarthritis of the second metatarsophalangeal joint [15] and patellofemoral pain syndrome [16].

There is one problem to overcome when using the 3D scanning and printing techniques for hallux valgus. We scanned participants' feet in a non-weight bearing position to scan the plantar areas of the foot. Considering that hallux valgus causes difficulty in walking, it would be better to design toe spreaders with the participants in a standing position. Gong et al showed that the HVA decreased in a weight-bearing position compared to a non-weight-bearing position [17]. The toe spreaders would have needed to be wider to correct the hallux valgus deformity if the foot had been scanned in a weight-bearing position.

A patient's HVA can increase over time, which will be incompatible with a customized toe spreader. A customized toe spreader need not be made from the beginning; our 3D printed personalized toe spreader can be easily reproduced by modifying the stored 3D modelling file.

The total amount of time to make a 3D printed personalized toe spreader was approximately seven hours. This was faster than the elapsed time to make the customized toe spreaders; several days to a week.

However, considering that cheaper 3D printers and materials are being developed at a breakneck pace, these costs will decrease soon. There were no side effects including skin rash and dermatitis during the period the toe spreaders were worn.

The total cost for scanning, image fusion, modelling, and materials used was approximately 50 dollars. This was cheaper than the cost of the customized toe spreaders (approximately 150 dollars). However, we did not include the cost for using the Objet500 Connex1 3D printer because this cost (approximately 200 dollars) was supported by the National Research Funds. The cost of our 3D printed toe spreader would have been approximately 250 dollars, which is more expensive than the customized toe spreaders available in our country. Considering that 3D printing materials are being developed and introduced rapidly, the cost of 3D printed materials will decrease soon and this personalized 3D printed toe spreader will be a good replacement in the orthosis market.

This study was not conducted as a randomized controlled design, which is the limitation of our study. Although there was no control group, the cost, time spent making the device, and capability to easily modify the orthosis design are the strong points of 3D printed toe spreaders compared to ready-made silicone toe spreaders or custom-made toe spreaders.

\section{Conclusions}

The 3D printer is a revolutionary tool that will change many things in the medical field. Our study provides one example (orthosis for hallux valgus) of these forthcoming changes and proved that personalized toe spreaders improved hallux valgus, with advantageous in terms of cost and time. If automated design software which used in study by Cha et al will be made, We can serve personalized toe spreader for many patients. And the development of 3D printer and matereals should make many patients they can choose the more something that they want. This suggests that the commercial use of 3D printed customized toe spreaders for hallux valgus may be possible.

\section{Author Disclosure Statement}

No competing financial interests exist. There was no commercial party involved in this study.

\section{Acknowledgements}

This work was supported by the ICT R\&D program of MSIP/IIPT [Grant No. B0101-17-1081] and by the Korea Institute for Advancement of Technology [Grant No. R0004587].

\section{References}

[1] D. B. Jones, R. Sung, C. Weinberg, T. Korelitz, R. Andrews, "Three-Dimensional Modeling May Improve Surgical Education and Clinical Practice," Surgical Innovation, vol. 23, no 2, pp. 189-195, 2016.

[2] E. Kroger, M. Dekiff, D. Dirksen, "3D printed simulation models based on real patient situations for hands-on practice," European Journal of Dental Education, vol. 21, no 4, 2016. 
[3] O. Al Jabbari, W. K. Abu Saleh, A. P. Patel, S. R. Igo, M. J. Reardon, "Use of three-dimensional models to assist in the resection of malignant cardiac tumors," Journal of Cardiac Surgery, vol. 31, pp. 581-583, 2016.

[4] M. J. Pfaff, D. M. Steinbacher, "Plastic Surgery Applications Using Three-Dimensional Planning and Computer-Assisted Design and Manufacturing," Plastic and Reconstructive Surgery, vol. 137, pp. 603e-616e, 2016.

[5] V. Mironov, V. Kasyanov, R. R. Markwald, "Organ printing: from bioprinter to organ biofabrication line," Current Opinion in Biotechnology, vol. 22, pp. 667-673, 2011.

[6] S. V. Murphy, A. Atala, "3D bioprinting of tissues and organs," Nature Biotechnology, vol. 32, pp. 773785, 2014.

[7] J. Zuniga, D. Katsavelis, J. Peck, J. Stollberg, M. Petrykowski, A. Carson, C. Fernandez, "Cyborg beast: a low-cost 3d-printed prosthetic hand for children with upper-limb differences," $B M C$ Research Notes, vol. 8, pp. 10, 2015.

[8] J. M. Zuniga, A. M. Carson, J. M. Peck, T. Kalina, R. M. Srivastava, K. Peck, "The development of a low-cost three-dimensional printed shoulder, arm, and hand prostheses for children," Prosthetics and Orthotics International, 2016.

[9] C. E. Dombroski, M. E. Balsdon, A. Froats, "The use of a low cost 3D scanning and printing tool in the manufacture of custom-made foot orthoses: a preliminary study," BMC Research Notes, vol. 7, pp. 443, 2014.

[10] J. V. Vanore, J. C. Christensen, S. R. Kravitz, J. M. Schuberth, J. L. Thomas, L. S. Weil, H. J. Zlotoff, R. W. Mendicino, S. D. Couture, "Clinical Practice Guideline First Metatarsophalangeal Joint Disorders Panel of the American College of F, Ankle S. Diagnosis and treatment of first metatarsophalangeal joint disorders. Section 1: Hallux valgus," Journal of Foot and Ankle Surgery, vol. 42, pp. 112-123, 2003.

[11] E. M. Roos, S. Brandsson, J. Karlsson, "Validation of the foot and ankle outcome score for ankle ligament reconstruction," Foot \& Ankle International, vol. 22, pp. 788-794, 2001.

[12] P. M. Kluding, K. Dunning, M. W. O'Dell, S. S. Wu, J. Ginosian, J. Feld, K. McBride, "Foot drop stimulation versus ankle foot orthosis after stroke: 30-week outcomes," Stroke, vol. 44, pp. 1660-1669, 2013.

[13] S. F. Tang, C. P. Chen, J. L. Pan, J. L. Chen, C. P. Leong, N. K. Chu, "The effects of a new foot-toe orthosis in treating painful hallux valgus," Archives of Physical Medicine and Rehabilitation, vol. 83, pp. 1792-1795, 2002.

[14] R. Schuh, M. Willegger, J. Holinka, R. Ristl, R. Windhager, A. H. Wanivenhaus, "Angular correction and complications of proximal first metatarsal osteotomies for hallux valgus deformity," International Orthopaedics, vol. 37, pp. 1771-1780, 2013.

[15] J. S. Lee, J. M. Hong, J. W. Jung, J. H. Shim, J. H. Oh, D. W. Cho, "3D printing of composite tissue with complex shape applied to ear regeneration," Biofabrication, vol. 6, pp. 024103, 2014.

[16] D. Kaya, O. A. Atay, M. J. Callaghan, A. Cil, O. Caglar, S. Citaker, I. Yuksel, M. N. Doral, "Hallux valgus in patients with patellofemoral pain syndrome," Knee Surgery, Sports Traumatology, Arthroscopy, vol. 17, pp. 1364-1367, 2009.

[17] H. Gong, Z. C. Sang, J. M. Wen, W. D. Sun, H. W. Hu, Y. C. Zhang, J. G. Zuo, H. X. Wang, "Correlative analysis on metatarsalgia and the X-ray measurement indexes under weight-bearing and nonweight-bearing of hallux valgus," Zhongguo Gu Shang, vol. 27, pp. 303-307, 2014. 